\title{
Stretching to meet needs: integrin-linked kinase and the cardiac pump
}

\author{
Deepak Srivastava ${ }^{1}$ and Sangho Yu \\ Gladstone Institute of Cardiovascular Disease and Department of Pediatrics, University of California at San Francisco, \\ San Francisco, California 94158, USA
}

In an elegant set of genetic and biochemical experiments reported in this issue of Genes \& Development, Bendig et al. (2006) show that integrin-linked kinase (ILK) is a critical component of the cardiac stretch sensor. Their findings link the cell surface and actin cytoskeleton to central signaling pathways. Here we discuss the significance of this work in light of other recent discoveries on the molecular mechanisms of cardiac stretch sensing.

The heart has the extraordinary capability of responding to physiologic demand by rapidly altering the amount of blood pumped with each heartbeat. Similarly, in response to pathologic conditions, the heart expands to increase cardiac output, although continued expansion eventually diminishes its ability to pump effectively. Widely known as the Frank-Starling curve, this property was described by Otto Frank and Ernest Henry Starling in 1918 (Fig. 1; Starling 1918). The molecular and cellular basis for rapid alteration in the contractility of heart muscle has attracted intense scientific interest focusing on the ability of the myocardium to sense "stretch" and respond by increasing strength of contraction and hypertrophy of individual myocytes.

The primary function of the heart is to supply oxygenated blood to peripheral tissues to meet metabolic demands. Heart failure-the leading cause of morbidity and mortality in the industrialized countries-occurs when cardiac output cannot meet metabolic requirements (Molkentin and Dorn 2001). Although the etiology and manifestations of heart failure are varied, a common early sign is pathological hypertrophic remodeling of the left ventricle (Tarone and Lembo 2003). In cardiac hypertrophy, cardiomyocytes increase in size by adding more sarcomeres to myofibrils to normalize myocardial wall stress from hemodynamic overload. Through complex and unclear mechanisms, persistent cardiac hypertrophy often leads to decreased contractility and dilated cardiomyopathy.

Cardiac hypertrophy is triggered by mechanical stretching of cardiomyocytes and release of neurohumoral factors, such as angiotensin II and epinephrine in response to pressure overload (Wakatsuki et al. 2004).

${ }^{1}$ Corresponding author.

E-MAIL dsrivastava@gladstone.ucsf.edu; FAX (415) 355-0141.

Article is online at http://www.genesdev.org/cgi/doi/10.1101/gad.1472506.
Mechanical stretching also promotes the release of autocrine and paracrine factors by cardiomyocytes and fibroblasts that activate hypertrophic and contractility signaling pathways (Sadoshima and Izumo 1997; Hunter and Chien 1999; Tarone and Lembo 2003). Ultimately, these pathways lead to the expression of stretch-responsive genes such as atrial natriuretic factor (anf) and vascular endothelial growth factor (vegf) (Pan et al. 1999; Seko et al. 1999; Franchini et al. 2000; Molkentin and Dorn 2001; Domingos et al. 2002; Kim et al. 2002; Zhang et al. 2003). Vegf transcription is stimulated by PKB/Akt and increases calcium transients that result in greater cardiac contractility (Rottbauer et al. 2005). These signaling pathways have been studied extensively by many groups in an effort to develop therapies for hypertrophic remodeling (Frey et al. 2004), but only recently have we began to understand how mechanical stretching is sensed by cardiomyocytes.

\section{Sarcomeric molecules in mechanotransduction}

In striated muscle cells, the sarcomere is the functional unit of contraction. Its main components are interdigitated actin and myosin filaments and their associated proteins. Mutations of several sarcomeric components in human and model animals have been associated with dilated cardiomyopathy or skeletal muscle myopathy (Seidman and Seidman 2001). These include cardiac troponins $\mathrm{T}$ and $\mathrm{I}$, titin, cardiac myosin-binding protein- $\mathrm{C}$, cardiac $\beta$-myosin heavy chain, myosin light chain, and tropomyosin. Mutations in sarcomeric genes cause structural disarray and altered physiology in cardiomyocytes.

Insight into the involvement of sarcomeric proteins in cardiac stretch sensing came from ablation of muscle LIM domain protein (MLP) in mice. Like mice with other sarcomeric protein mutations, mice lacking MLP displayed sarcomeric disarray, contractile dysfunction, and cardiac dilatation (Arber et al. 1997). However, before dilated cardiomyopathy developed, the mechanical stretch response was impaired, while hypertrophic signaling triggered by humoral factors, such as phenylephrine or endothelin-1, was intact (Knoll et al. 2002). Since it is associated with the Z-disc linking $\alpha$-actinin to titincap (T-cap) and is highly expressed in the heart, MLP 


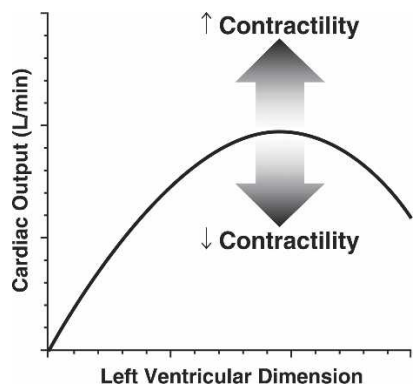

Figure 1. Frank-Starling curve. The Frank-Starling principle describes the relationship between stroke volume (cardiac output) and left ventricular end-diastolic volume or dimension (preload). Increased preload causes the stretch of cardiomyocytes, which results in increased force generation and, consequently, increased cardiac output. Thus, when the heart rate is constant, cardiac output is directly related to preload. However, at a certain point, cardiac output will not increase with any further increase in preload, but will decrease instead. An increase or decrease in cardiac contractility at a given end-diastolic volume shifts the curve up or down, respectively.

might help to maintain the cytoarchitecture of cardiomyocytes and to sense mechanical stretching (Fig. 2; Arber et al. 1997; Knoll et al. 2002). In humans, a missense mutation in human MLP was associated with dilated cardiomyopathy (Knoll et al. 2002), indicating that sarcomeric proteins may be essential components of the cardiac mechanical sensor, particularly ones closely associated with Z-discs that connect the intracellular contractile machinery to the extracellular environment through a structure called the costamere (Fig. 2).

\section{Costameres: where the contractile apparatus meets the extracellular matrix (ECM)}

Costameres link the Z-disc to the sarcolemma membrane and consist of a complex protein network of cytoskeletal proteins and signaling kinases (Samarel 2005). Because they connect the intracellular contractile apparatus to the extracellular environment and are associated with many signaling molecules, costameres are uniquely positioned to sense mechanical stretch and transduce downstream signals.

Integrins are heterodimeric integral membrane proteins connecting the ECM to the intracellular actin cytoskeleton and part of the costameric network in cardiomycytes (Sussman et al. 2002). The cytoplasmic domains of integrins, particularly $\beta$-subunits, interact with adaptor proteins (e.g., vinculin, talin, and $\alpha$-actinin) that are critical for transmitting mechanical force between the ECM and the actin cytoskeleton (Fig. 2; Samarel 2005). In addition to their structural role, integrins and their associated adaptor proteins recruit signaling molecules to the membrane that are important for the survival and growth of cardiomyocytes. These molecules include focal adhesion kinase, proline-rich tyrosine kinase 2, Src, c-Src kinase, ILK, protein kinase C (PKC), and p21-acti- vated kinase (PAK) (Samarel 2005). Furthermore, the focal adhesion complex may be linked to growth factor receptors (Eliceiri 2001), indicating a potential mechanism for cross-talk between mechanotransduction and growth factor-mediated hypertrophic and contractility signals.

The importance of integrins in cardiac function was revealed in two in vivo studies of genetically modified mice. In one, transgenic mice expressing a dominantnegative form of $\beta$-integrin specifically in the heart (Keller et al. 2001) died perinatally with extensive fibrosis. Those that survived to adulthood developed spontaneous dilated cardiomyopathy with defective contractility and relaxation. Consistent with this finding, in the other study, inactivation of the $\beta 1$-integrin gene only in the ventricles with a Cre-loxP system also resulted in dilated cardiomyopathy with increased fibrosis, indicating an important role of integrins in cardiac function and homeostasis (Shai et al. 2002). Nevertheless, exactly how integrins sense mechanical stress is not understood.

A clue came from the targeted deletion of melusin in mice (Brancaccio et al. 2003). Melusin is specifically expressed in striated muscle, enriched in costameres, and interacts with the cytoplasmic domain of $\beta 1$-integrin (Brancaccio et al. 1999). Under physiologic conditions, melusin-null mice showed normal cardiac structure, but in response to pressure overload, they failed to activate stretch-responsive genes and developed dilated cardio-

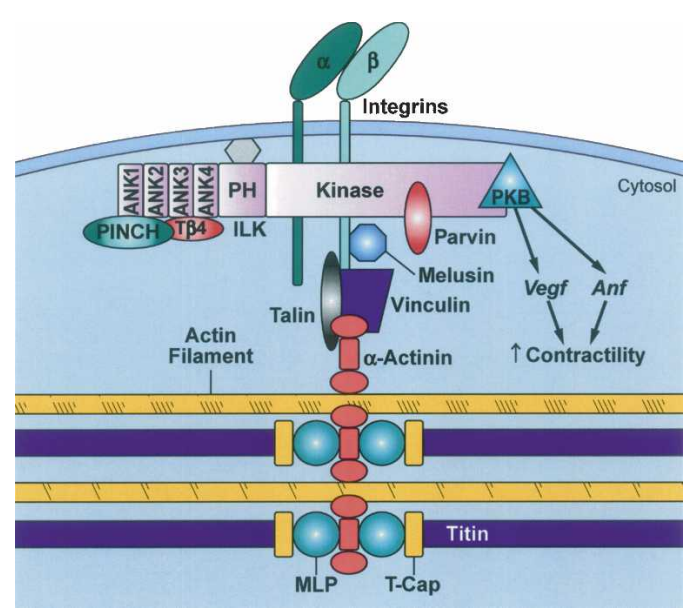

Figure 2. Structure of costamere and Z-disc. $\alpha$-Actinin binds to actin and MLP-T-cap-titin complex to organize the cytoarchitecture and to talin and vinculin to connect the contractile machinery to integrins and the ECM. The cytoplasmic domain of $\beta$-integrin binds ILK and melusin, and ILK plays both structural and functional roles at the costamere by recruiting various adaptor molecules, including PINCH and parvins, and by phosphorylating PKB and other signaling molecules. In this issue of Genes \& Development, Bendig et al. (2006) demonstrate that ILK is a novel component of the cardiac mechanical stretch sensor and activates PKB to regulate the response to stretch in fish. White et al. (2006) also demonstrate the requirement of ILK for cardiac function and PKB activation in the mouse. Some components of the costamere or Z-disc are omitted for clarity. ILK is shown with its distinct domains. (T $\beta 4)$ Thymosin $\beta 4$. 
myopathy (Brancaccio et al. 2003). As in MLP mutant mice, however, ablation of melusin did not affect the development of cardiac hypertrophy triggered by humoral factors in the absence of mechanical stress, indicating that melusin might be solely involved in the hypertrophic response to mechanical stress. Significantly, unlike MLP-null mice, melusin-null mice showed no defects of cardiac structure or function under normal physiologic conditions, suggesting that melusin's role is more functional than structural. Interestingly, cardiac overexpression of melusin induced mild cardiac hypertrophy under basal physiologic conditions but protected the heart from dilated cardiomyopathy after prolonged pressure overload (De Acetis et al. 2005). Under pressure overload, these transgenic mice developed concentric cardiac hypertrophy without apparent cardiomyocyte apoptosis and fibrosis, indicating that melusin might be involved in the activation of an adaptive hypertrophic and contractility program.

\section{ILK is a novel component of the cardiac mechanical stretch sensor}

Bendig et al. (2006) add one more piece to the puzzle of cardiac stretch sensing by focusing on ILK (Fig. 2). The authors studied a recessive zebrafish mutant, derived through ethylnitrosourea (ENU)-induced mutagenesis, that develops embryonic heart failure. This mutant, called main squeeze ( $m s q)$, develops progressive reduction of cardiac contractility and severe down-regulation of the stretch-responsive genes anf and vegf. However, cardiac morphogenesis and sarcomeric structure are nor$\mathrm{mal}$ in $m s q$ mutant embryos. By positional cloning, Bendig et al. found that the $m s q$ phenotype is caused by a homozygous missense mutation in the zebrafish ilk gene (zilk), which converts a highly conserved leucine to proline (L308P).

ILK consists of four $\mathrm{N}$-terminal ankyrin repeats, a pleckstrin homology $(\mathrm{PH})$ domain, and a C-terminal kinase domain (Fig. 2; Legate et al. 2006); the msq mutation occurred in the kinase domain. In the zebrafish heart, ILK is enriched in the costameres and Z-discs, making it a good candidate for a component of the cardiac mechanical stretch sensor (Bendig et al. 2006). Injecting morpholino-modified antisense oligonucleotide against zilk mRNA (MO-zilk) into one-cell zebrafish embryos reproduced the $m s q$ heart phenotype, including lower expression of anf and vegf, and injecting wild-type zilk mRNA into the ms $q$ embryos rescued the $m s q$ phenotype. Interestingly, injection of human wild-type ilk mRNA also rescued the $m s q$ phenotype, providing convincing evidence that the function of ILK in maintaining cardiac contractility is conserved between zebrafish and human.

ILK interacts with numerous cytoskeletal, adaptor and signaling molecules through its $\mathrm{N}$-terminal ankyrin-repeat domain and C-terminal kinase domain (Legate et al. 2006). The ankyrin-repeat domain binds to PINCH (particularly interesting Cys-His-rich protein) and ILK-associated phosphatase (Tu et al. 1999; Legate et al. 2006); the kinase domain of ILK interacts with the cytoplasmic domain of $\beta$-integrins, $\alpha$-parvin/actopaxin/CH-ILKBP, $\beta$-parvin/affixin, paxillin, PI3K-dependent kinase-1, and protein kinase B (PKB/AKT) (Fig. 2; Hannigan et al. 1996; Nikolopoulos and Turner 2000, 2001; Persad et al. 2001; Tu et al. 2001; Yamaji et al. 2001; Pasquet et al. 2002). These interactions link ILK to the actin cytoskeleton and growth factor receptors, making it a crucial component of the focal adhesion complex both as an adaptor and a signaling kinase. The role of ILK as a kinase, especially for the phosphorylation of PKB at Ser 473, has been controversial because some crucial residues in the $\mathrm{ki}$ nase domain are either missing or not conserved, compared with canonical kinase sequences (Legate et al. 2006). However, ILK appears to directly bind PKB and phosphorylate Ser 473 in vitro, even though other kinases also seem to be involved in PKB phosphorylation at Ser 473 (Persad et al. 2001).

Through in vitro kinase and immunoprecipitation assays, Bendig et al. (2006) showed that the msq mutation disrupts ILK kinase activity and its ability to bind $\beta$-parvin, but not paxillin. Injecting kinase-deficient human ILK (Persad et al. 2001) mRNA into msq embryos failed to rescue the $m s q$ heart phenotype, implying that the reduced kinase activity of ILK $^{\mathrm{L} 308 \mathrm{P}}$ might cause the progressive loss of cardiac contractility in $m s q$ mutant embryos. Constitutively active PKB and wild-type Vegf, but not PKB lacking kinase activity, partially rescued the $m s q$ heart phenotype, strongly supporting their hypothesis that an ILK-PKB-VEGF pathway might be crucial for cardiac mechanosensing and mechanotransduction and the maintenance of cardiac function. Also, there is evidence that the ILK-PKB pathway might regulate anf transcription, further supporting the role of ILK in cardiac stretch sensing (Morisco et al. 2000).

Impaired interaction between ILK $^{\mathrm{L} 308 \mathrm{P}}$ and $\beta$-parvin also accounts for the $m s q$ heart phenotype. Another mutant form of ILK (hILK ${ }^{\mathrm{E} 359 \mathrm{~K}}$ ) with dramatically reduced binding to $\beta$-parvin (Yamaji et al. 2001) failed to rescue the $m s q$ phenotype, and conversely, injection of a morpholino-modified antisense oligonucleotide against $\beta$-parvin into the wild-type embryos phenocopied the ms $q$ heart phenotype (Bendig et al. 2006). However, injection of an antisense oligonucleotide against another ILK-binding molecule, paxillin, did not cause heart failure, emphasizing the specific role of $\beta$-parvin in the maintenance of cardiac function. Through powerful genetic tools, this body of work convincingly shows that ILK is crucial for cardiac stretch sensing and contractility in zebrafish.

Also in this issue of Genes \& Development, White et al. (2006) show that ILK is necessary for normal cardiac function in mice. Although homozygous ILK-null mice die at the peri-implantation stage (Sakai et al. 2003), cardiac-specific deletion of ILK resulted in spontaneous dilated cardiomyopathy wihtin weeks after birth. While some features of ILK disruption were similar in fish and mice, including cardiac dysfunction and decreased PKB activation, mice lacking ILK also had disaggregation of cardiomyocytes and cardiac fibrosis. Whether ILK func- 
tions as a critical component of the stretch-sensing machinery in the mouse heart remains to be determined.

\section{Questions and future perspectives}

Along with melusin, ILK is the only costameric component that, when mutated, affects cardiac function without causing structural disarray of cardiomyocytes. This is a key finding for understanding the process of cardiac hypertrophy and subsequent cardiac failure and for developing strategies to tackle this epidemic disorder. However, we should be cautious about interpreting the data of Bendig et al. (2006) and in applying this knowledge to future therapeutic purposes before the exact mechanism is fully understood. As with most good studies, this one raises several important questions.

First, both melusin and ILK bind the cytoplasmic domain of $\beta$-integrin and seem to act in the cardiac stretchsensing process. Moreover, in the melusin-overexpressing mouse heart, anf expression is increased, and $\mathrm{PKB}$ and extracellular signal regulated kinase (ERK) 1/2 are hyperphosphorylated (De Acetis et al. 2005). Thus, it will be interesting to test whether melusin interacts with ILK and transmits downstream signals through ILK.

Second, the R211A mutation of ILK lacks kinase activity and is commonly used to study kinase function in ILK (Persad et al. 2001). However, some studies suggest that the same mutation also disrupts ILK binding to $\alpha$-parvin (Attwell et al. 2003), which is highly homologous to $\beta$-parvin, and that the kinase activity of ILK is required for its binding to $\beta$-parvin (Yamaji et al. 2001). As a result, the reduced binding of $m s q$ mutant $z \mathrm{ILK}^{\mathrm{L} 308 \mathrm{P}}$ to $\beta$-parvin might be due to the reduced kinase activity of the mutant protein. By the same token, the $\beta$-parvinbinding-deficient E359K mutation of ILK has significantly reduced kinase activity, although there are discrepancies in reports from different groups (Novak et al. 1998; Persad et al. 2001; Yamaji et al. 2001). Because of this close relationship between the kinase activity and the ability of ILK to bind other proteins, it is difficult to conclude whether the msq heart phenotype is caused by impaired kinase activity or the reduced binding to $\beta$-parvin, or both.

Finally, ILK is an integral component of the focal adhesion complex that is important for cell attachment and migration and for cell proliferation, differentiation, and survival (Guo and Wu 2002; Miller et al. 2003). Interaction of ILK with the G-actin-sequestering protein thymosin $\beta-4$ promotes cardiac cell migration and protection from hypoxia (Bock-Marquette et al. 2004). In worms and flies, deletion of ilk results in muscle cell detachment from the basal membrane (Zervas et al. 2001; Mackinnon et al. 2002). Therefore, it is surprising that $m s q$ mutants do not display defects in cell proliferation, differentiation, survival, or attachments. However, this may reflect the limited repertoire of cardiac stresses that can be generated in fish, as mice lacking ILK in the heart do display defects of cell attachment (White et al. 2006). Given the central role of ILK, future mecha- nistic studies could prove very important for human heart disease.

\section{Acknowledgments}

We thank S. Ordway and G. Howard for editorial assistance. D.S. was supported by grants from NHLBI/NIH, March of Dimes Birth Defects Foundation and is an Established Investigator of the American Heart Association.

\section{References}

Arber, S., Hunter, J.J., Ross Jr., J., Hongo, M., Sansig, G., Borg, J., Perriard, J.C., Chien, K.R., and Caroni, P. 1997. MLP-deficient mice exhibit a disruption of cardiac cytoarchitectural organization, dilated cardiomyopathy, and heart failure. Cell 88: 393-403.

Attwell, S., Mills, J., Troussard, A., Wu, C., and Dedhar, S. 2003. Integration of cell attachment, cytoskeletal localization, and signaling by integrin-linked kinase (ILK), CH-ILKBP, and the tumor suppressor PTEN. Mol. Biol. Cell 14: 4813-4825.

Bendig, G., Grimmler, M., Huttner, I.G., Wessels, G., Dahme, T., Just, S., Trano, N., Katus, H.A., Fishman, M.C., and Rottbauer, W. 2006. Integrin-linked kinase, a novel component of the cardiac mechanical stretch sensor, controls contractility in the zebrafish heart. Genes \& Dev. (this issue).

Bock-Marquette, I., Saxena, A., White, M.D., Dimaio, J.M., and Srivastava, D. 2004. Thymosin $\beta 4$ activates integrin-linked kinase and promotes cardiac cell migration, survival and cardiac repair. Nature 432: 466-472.

Brancaccio, M., Guazzone, S., Menini, N., Sibona, E., Hirsch, E., De Andrea, M., Rocchi, M., Altruda, F., Tarone, G., and Silengo, L. 1999. Melusin is a new muscle-specific interactor for $\beta(1)$ integrin cytoplasmic domain. J. Biol. Chem. 274: 29282-29288.

Brancaccio, M., Fratta, L., Notte, A., Hirsch, E., Poulet, R., Guazzone, S., De Acetis, M., Vecchione, C., Marino, G., Altruda, F., et al. 2003. Melusin, a muscle-specific integrin $\beta 1$-interacting protein, is required to prevent cardiac failure in response to chronic pressure overload. Nat. Med. 9: 68-75.

De Acetis, M., Notte, A., Accornero, F., Selvetella, G., Brancaccio, M., Vecchione, C., Sbroggio, M., Collino, F., Pacchioni, B., Lanfranchi, G., et al. 2005. Cardiac overexpression of melusin protects from dilated cardiomyopathy due to longstanding pressure overload. Circ. Res. 96: 1087-1094.

Domingos, P.P., Fonseca, P.M., Nadruz Jr., W., and Franchini, K.G. 2002. Load-induced focal adhesion kinase activation in the myocardium: Role of stretch and contractile activity. Am. J. Physiol. Heart Circ. Physiol. 282: H556-H564.

Eliceiri, B.P. 2001. Integrin and growth factor receptor crosstalk. Circ. Res. 89: 1104-1110.

Franchini, K.G., Torsoni, A.S., Soares, P.H., and Saad, M.J. 2000. Early activation of the multicomponent signaling complex associated with focal adhesion kinase induced by pressure overload in the rat heart. Circ. Res. 87: 558-565.

Frey, N., Katus, H.A., Olson, E.N., and Hill, J.A. 2004. Hypertrophy of the heart: A new therapeutic target? Circulation 109: 1580-1589.

Guo, L. and Wu, C. 2002. Regulation of fibronectin matrix deposition and cell proliferation by the PINCH-ILK-CH-ILKBP complex. FASEB J. 16: 1298-1300.

Hannigan, G.E., Leung-Hagesteijn, C., Fitz-Gibbon, L., Coppolino, M.G., Radeva, G., Filmus, J., Bell, J.C., and Dedhar, S. 1996. Regulation of cell adhesion and anchorage-dependent 
growth by a new $\beta 1$-integrin-linked protein kinase. Nature 379: 91-96.

Hunter, J.J. and Chien, K.R. 1999. Signaling pathways for cardiac hypertrophy and failure. N. Engl. I. Med. 341: 12761283.

Keller, R.S., Shai, S.Y., Babbitt, C.J., Pham, C.G., Solaro, R.J., Valencik, M.L., Loftus, J.C., and Ross, R.S. 2001. Disruption of integrin function in the murine myocardium leads to perinatal lethality, fibrosis, and abnormal cardiac performance. Am. J. Pathol. 158: 1079-1090.

Kim, C.H., Cho, Y.S., Chun, Y.S., Park, J.W., and Kim, M.S. 2002. Early expression of myocardial HIF- $1 \alpha$ in response to mechanical stresses: Regulation by stretch-activated channels and the phosphatidylinositol 3-kinase signaling pathway. Circ. Res. 90: E25-E33.

Knoll, R., Hoshijima, M., Hoffman, H.M., Person, V., LorenzenSchmidt, I., Bang, M.L., Hayashi, T., Shiga, N., Yasukawa, H., Schaper, W., et al. 2002. The cardiac mechanical stretch sensor machinery involves a $\mathrm{Z}$ disc complex that is defective in a subset of human dilated cardiomyopathy. Cell 111: 943955.

Legate, K.R., Montanez, E., Kudlacek, O., and Fassler, R. 2006. ILK, PINCH and parvin: The tIPP of integrin signalling. Nat. Rev. Mol. Cell Biol. 7: 20-31.

Mackinnon, A.C., Qadota, H., Norman, K.R., Moerman, D.G., and Williams, B.D. 2002. C. elegans PAT-4/ILK functions as an adaptor protein within integrin adhesion complexes. Curr. Biol. 12: 787-797.

Miller, M.G., Naruszewicz, I., Kumar, A.S., Ramlal, T., and Hannigan, G.E. 2003. Integrin-linked kinase is a positive mediator of L6 myoblast differentiation. Biochem. Biophys. Res. Commun. 310: 796-803.

Molkentin, J.D. and Dorn II, I.G. 2001. Cytoplasmic signaling pathways that regulate cardiac hypertrophy. Annu. Rev. Physiol. 63: 391-426.

Morisco, C., Zebrowski, D., Condorelli, G., Tsichlis, P., Vatner, S.F., and Sadoshima, J. 2000. The Akt-glycogen synthase kinase $3 \beta$ pathway regulates transcription of atrial natriuretic factor induced by $\beta$-adrenergic receptor stimulation in cardiac myocytes. J. Biol. Chem. 275: 14466-14475.

Nikolopoulos, S.N. and Turner, C.E. 2000. Actopaxin, a new focal adhesion protein that binds paxillin LD motifs and actin and regulates cell adhesion. J. Cell Biol. 151: 1435-1448.

- 2001. Integrin-linked kinase (ILK) binding to paxillin LD1 motif regulates ILK localization to focal adhesions. J. Biol. Chem. 276: 23499-23505.

Novak, A., Hsu, S.C., Leung-Hagesteijn, C., Radeva, G., Papkoff, J., Montesano, R., Roskelley, C., Grosschedl, R., and Dedhar, S. 1998. Cell adhesion and the integrin-linked kinase regulate the LEF-1 and $\beta$-catenin signaling pathways. Proc. Nat1. Acad. Sci. 95: 4374-4379.

Pan, J., Fukuda, K., Saito, M., Matsuzaki, J., Kodama, H., Sano, M., Takahashi, T., Kato, T., and Ogawa, S. 1999. Mechanical stretch activates the JAK/STAT pathway in rat cardiomyocytes. Circ. Res. 84: 1127-1136.

Pasquet, J.M., Noury, M., and Nurden, A.T. 2002. Evidence that the platelet integrin $\alpha I I b \beta 3$ is regulated by the integrinlinked kinase, ILK, in a PI3-kinase dependent pathway. Thromb. Haemost. 88: 115-122.

Persad, S., Attwell, S., Gray, V., Mawji, N., Deng, J.T., Leung, D., Yan, J., Sanghera, J., Walsh, M.P., and Dedhar, S. 2001. Regulation of protein kinase B/Akt-serine 473 phosphorylation by integrin-linked kinase: Critical roles for kinase activity and amino acids arginine 211 and serine 343. J. Biol. Chem. 276: 27462-27469.

Rottbauer, W., Just, S., Wessels, G., Trano, N., Most, P., Katus,
H.A., and Fishman, M.C. 2005. VEGF-PLC $\gamma 1$ pathway controls cardiac contractility in the embryonic heart. Genes \& Dev. 19: 1624-1634.

Sadoshima, J. and Izumo, S. 1997. The cellular and molecular response of cardiac myocytes to mechanical stress. Annu. Rev. Physiol. 59: 551-571.

Sakai, T., Li, S., Docheva, D., Grashoff, C., Sakai, K., Kostka, G., Braun, A., Pfeifer, A., Yurchenco, P.D., and Fassler, R. 2003. Integrin-linked kinase (ILK) is required for polarizing the epiblast, cell adhesion, and controlling actin accumulation. Genes \& Dev. 17: 926-940.

Samarel, A.M. 2005. Costameres, focal adhesions, and cardiomyocyte mechanotransduction. Am. J. Physiol. Heart Circ. Physiol. 289: H2291-H2301.

Seidman, J.G. and Seidman, C. 2001. The genetic basis for cardiomyopathy: From mutation identification to mechanistic paradigms. Cell 104: 557-567.

Seko, Y., Takahashi, N., Shibuya, M., and Yazaki, Y. 1999. Pulsatile stretch stimulates vascular endothelial growth factor (VEGF) secretion by cultured rat cardiac myocytes. Biochem. Biophys. Res. Commun. 254: 462-465.

Shai, S.Y., Harpf, A.E., Babbitt, C.J., Jordan, M.C., Fishbein, M.C., Chen, J., Omura, M., Leil, T.A., Becker, K.D., Jiang, M., et al. 2002. Cardiac myocyte-specific excision of the $\beta 1$ integrin gene results in myocardial fibrosis and cardiac failure. Circ. Res. 90: 458-464.

Starling, E.H. 1918. Linacre lecture on the law of the heart. Longmans, Green and Co., London, UK.

Sussman, M.A., McCulloch, A., and Borg, T.K. 2002. Dance band on the Titanic: Biomechanical signaling in cardiac hypertrophy. Circ. Res. 91: 888-898.

Tarone, G. and Lembo, G. 2003. Molecular interplay between mechanical and humoral signalling in cardiac hypertrophy. Trends Mol. Med. 9: 376-382.

Tu, Y., Li, F., Goicoechea, S., and Wu, C. 1999. The LIM-only protein $\mathrm{PINCH}$ directly interacts with integrin-linked kinase and is recruited to integrin-rich sites in spreading cells. Mol. Cell. Biol. 19: 2425-2434.

Tu, Y., Huang, Y., Zhang, Y., Hua, Y., and Wu, C. 2001. A new focal adhesion protein that interacts with integrin-linked kinase and regulates cell adhesion and spreading. J. Cell Biol. 153: $585-598$.

Wakatsuki, T., Schlessinger, J., and Elson, E.L. 2004. The biochemical response of the heart to hypertension and exercise. Trends Biochem. Sci. 29: 609-617.

White, D.E., Coutu, P., Shi, Y.-F., Tardif, J.-C., Nattel, S., St. Arnaud, R., Dedhar, S., and Muller, W.J. 2006. Targeted ablation of ILK from the murine heart results in dilated cardiomyopathy and spotaneous heart failure. Genes \& Dev. (this issue).

Yamaji, S., Suzuki, A., Sugiyama, Y., Koide, Y., Yoshida, M., Kanamori, H., Mohri, H., Ohno, S., and Ishigatsubo, Y. 2001. A novel integrin-linked kinase-binding protein, affixin, is involved in the early stage of cell-substrate interaction. $J$. Cell Biol. 153: 1251-1264.

Zervas, C.G., Gregory, S.L., and Brown, N.H. 2001. Drosophila integrin-linked kinase is required at sites of integrin adhesion to link the cytoskeleton to the plasma membrane. $I$. Cell Biol. 152: 1007-1018.

Zhang, S., Weinheimer, C., Courtois, M., Kovacs, A., Zhang, C.E., Cheng, A.M., Wang, Y., and Muslin, A.J. 2003. The role of the Grb2-p38 MAPK signaling pathway in cardiac hypertrophy and fibrosis. J. Clin. Invest. 111: 833-841. 


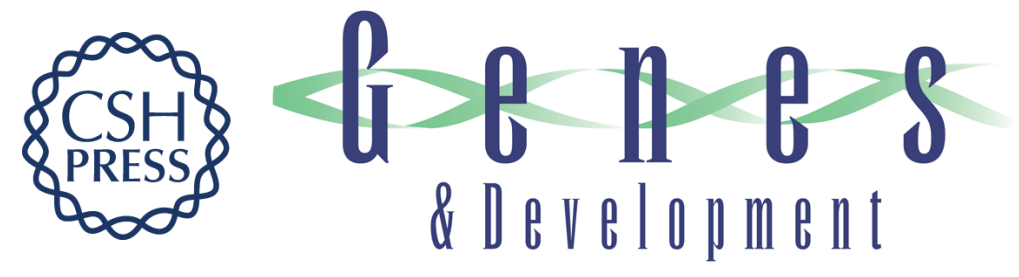

\section{Stretching to meet needs: integrin-linked kinase and the cardiac pump}

Deepak Srivastava and Sangho Yu

Genes Dev. 2006, 20:

Access the most recent version at doi:10.1101/gad.1472506

\section{Related Content}

References

\section{License}

Email Alerting Service
This article cites 42 articles, 20 of which can be accessed free at: http://genesdev.cshlp.org/content/20/17/2327.full.html\#ref-list-1

Articles cited in:

http://genesdev.cshlp.org/content/20/17/2327.full.html\#related-urls

Integrin-linked kinase, a novel component of the cardiac mechanical stretch sensor, controls contractility in the zebrafish heart

Garnet Bendig, Matthias Grimmler, Inken G. Huttner, et al.

Genes Dev. September, 2006 20: 2361-2372

Receive free email alerts when new articles cite this article - sign up in the box at the top right corner of the article or click here.

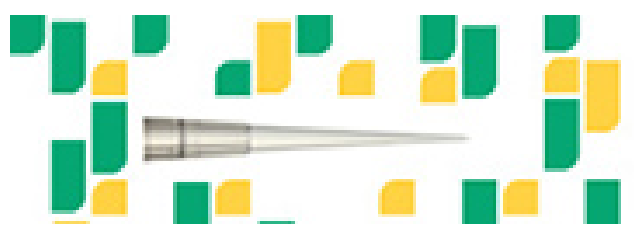

Focused on your science. 